\title{
Beating Psychosis to the Punch: The Treatment Options Debate
}

\author{
By Jack M. Gorman, MD
}

This month's CNS Spectrums features several articles on treating prodromal schizophrenia. As has been previously discussed in CNS Spectrums, this is currently a hot topic as clinicians and scientists have come to the conclusion that recently introduced antipsychotic medications are safer than older ones, and, therefore, we can be bolder in introducing them earlier in the course of schizophrenia.

The assumption, however, could turn out to be one of those things that is more what we wish is the case than what the data can actually support. Although, it has been almost 15 years since clozapine heralded the beginning of the "atypical" antipsychotic era in the pharmacologic treatment of schizophrenia, it is increasingly asked just how much better the atypicals are than the older, "typical" antipsychotic medications.

Judging the safety of a medication always entails measuring its efficacy. No risk is worth taking for a medication that offers no benefit. Increasing risk is acceptable, however, with increasing level of disease severity and effectiveness of the treatment. Thus, for usually fatal illnesses, such as pancreatic cancer or glioblastoma for example, measures that might produce severe adverse events are worth considering. Schizophrenia certainly qualifies as a severe and life-threatening illness. Hence, some level of adverse events is a reasonable trade-off for efficacy.

It is important to remember, however, that studies have rarely, if ever, shown that atypical antipsychotics have superior efficacy compared with typical antipsychotics, at least for treating the positive symptoms of schizophrenia. Haloperidol, a potent $D_{2}$ dopamine receptor antagonist, is hard to beat in that regard. In terms of negative and cognitive symptoms of schizophrenia, preclinical studies certainly suggest mechanisms by which atypicals ought to be superior to typicals. This includes evidence that atypicals stimulate serotonin $1 \mathrm{~A}$ receptors, block serotonin $2 \mathrm{~A}$ receptors, stimulate glutamate activity in the frontal cortex, and even increase hippocampal neurogenesis. Nevertheless, it remains a controversial subject whether the atypicals actually treat negative and cognitive symptoms better than typicals. If they do, the differences are certainly modest and of limited clinical significance. So far, the one distinction that stands out for the atypicals is the anti-suicide effect of clozapine. Unfortunately, this property does not seem to be shared by other drugs in the class.

If efficacy does not obviously separate the atypicals from the typicals, we can only rely on safety differences to determine whether one class is superior to the other. Without question, the risk of extrapyramidal effects and tardive dykinesia is far lower with the atypicals. On the other hand, weight gain and metabolic abnormalities have emerged as major risk factors for at least some of the atypicals. Physicians fear causing tardive dyskinesia, an unfortunate but non-fatal adverse event, but seem to tolerate obesity and the risk of type II diabetes mellitus in their patients - both associated with shortened lifespan. Patients, on the other hand, seem more bothered by weight gain than parkinsonism. Hence, the safety and tolerability benefits of the atypicals are not as clear-cut as some might assume.

In the National Institute of Mental Health-funded Clinical Antipsychotic Trials in Intervention Effectiveness examining the relative benefits of antipsychotic medications, the typical agent perphenazine is being studied along with most of the currently available atypicals. A few experts have quietly predicted that perphenazine just might come out on par with the atypicals. It is certainly a lot cheaper.

I want to make clear that I almost always prescribe atypical antipsychotics rather than typicals, and I teach my students and residents to do the same. Nevertheless, it is sobering to sit back and consider the available evidence. Sobering, but hopefully not dismaying. We have made significant advances in treating schizophrenia in the last decade, and the introduction of atypical antipsychotics has been part of that progress. Sometimes what we wish to be true, however, overwhelms what the available data allow us to assert with absolute confidence. CNS 